\title{
A GENERALIZATION OF A COMMUTATOR THEOREM OF MIKUSINSKI
}

\author{
E. C. PAIGE
}

1. Introduction. In a series of papers [4]-[6] Mikusinski and Sikorski considered the following problem. Let $V$ be a vector space over a field of characteristic 0 and let $D$ be a locally algebraic linear transformation of $V$ (i.e., given any $x$ in $V$ there is a polynomial $f(\lambda) \neq 0$ over $F$ with $x f(D)=0)$. If $A=F[\lambda]$ is the polynomial ring in one variable over $F, V$ becomes an $A$-module under the definition $x f(\lambda)=x f(D)$ for $x$ in $V, f(\lambda)$ in $A$. The Mikusinski-Sikorski hypotheses on $V$ and $D$ can be phrased as follows.

I. If $f(\lambda) \in A$ has degree $n \geqq 1$, the kernel of $f(D)$ has dimension $\leqq n$.

II. If $f(\lambda), g(\lambda)$ in $A$ have positive degrees and if the dimensions of $\operatorname{Ker} f(D)$ and $\operatorname{Ker} g(D)$ are $m$ and $n$ respectively, the dimension of $\operatorname{Ker} f(D) g(D)$ is $m+n$.

Mikusinski and Sikorski [5], [6] then proved the

THEOREM. If $D$ is a locally algebraic linear transformation of $V$ satisfying I and II, there is a linear transformation $T$ of $V$ with TD$D T=I$, the identity transformation of $V$.

Mikusinski [4] also demonstrated a converse; namely he proved the

THEOREM. If $D$ is a locally algebraic linear transformation of $V$ satisfying condition I and if there is a linear transformation $T$ of $V$ with $T D-D T=I$ then condition II is satisfied.

The generalizations treated in this paper may be formulated as follows. Let $D$ be a locally algebraic linear transformation of $V$; instead of the conditions listed above, the assumption will be

III. $V$ is a divisible $A$-module (i.e., given $y$ in $V$ and $f(\lambda) \neq 0$ in $A=F[\lambda]$ there is an $x$ in $V$ with $x f(\lambda)=x f(D)=y)$.

The first theorem may be stated as

THEOREM 1. If $D$ is a locally algebraic linear transformation of $V$ satisfying condition III, then a linear transformation, $T$, of $V$ exists with $T D-D T=I$.

The converse result established is

THEOREM 2. If $D$ is a locally algebraic linear transformation on $V$

Received by the editors April 11, 1966. 
over $F$ of characteristic 0 and if a linear transformation, $T$, of $V$ exists with $T D-D T=I$, then condition III is satisfied.

The characteristic 0 hypothesis cannot be omitted in Theorem 2 as will be shown by an example due to A. A. Albert. It will be shown that these results imply a generalization of the Mikusinski-Sikorski results and that this generalization implies one obtained by $\mathrm{Mr}$. James Geer in a Master's thesis [2] at the University of Virginia. The author expresses his appreciation to Professor M. Rosenblum for calling this problem to his attention.

2. Sufficiency of the condition III. As usual the $A$-module $V$ will be termed a primary $A$-module if there is an irreducible element $p=p(\lambda)$ of $A$ such that every element of $V$ is in the kernel of $(p(D))^{k}$ for some $k$. The following lemmas are well known [3] but are included for convenience.

Lemma 1. If $D$ is a locally algebraic linear transformation of $V$, then $V$ is the (weak) direct sum of primary A-modules.

Lemma 2. A direct sum of $A$-modules is divisible if and only if each summand is divisible.

Now if $V_{p}$ is a primary component of $V$ and if $T_{p}$ is a linear transformation on $V_{p}$ satisfying $\left[T_{p}, D\right]=T_{p} D-D T_{p}=I$ on $V_{p}$, then the direct sum $T=\sum_{p} T_{p}$ of the $T_{p}$ for $p$ ranging over the irreducible polynomials of $A$ clearly satisfies $[T, D]=I$ on $V$.

The previous remark and Lemmas 1 and 2 clearly reduce the problem to the case in which $V$ is a primary divisible A-module for the prime $p$ of $A$, and this hypothesis is maintained for the remainder of this section.

For each integer $k \geqq 1$ let

$$
V_{k}=\left\{x \in V: x(p(D))^{k}=0\right\}
$$

so that $V_{k}$ is the kernel of $(p(D))^{k}$, is an $A$-submodule of $V$ (i.e., is a $D$-invariant subspace of $V)$, and satisfies $V_{k} \subseteq V_{k+1}, V_{k+1}(p(D)) \subset V_{k}$, and $\bigcup_{k=1}^{\infty} V_{k}=V$.

Lemma 3. If $V$ is a primary divisible $A$-module for the prime polynomial $p(\lambda)$ of degree $m \geqq 1$, there is a basis $\left\{x(\alpha, k) D^{j}\right\}_{\alpha, j, k}$ of $V$ where $\alpha$ ranges over some index set, and $0 \leqq j \leqq m-1$ and $k \geqq 1$ is an integer.

Since $x p=x p(D)=0$ for all $x \in V_{1}, V_{1}$ is a vector space over the field $K=F[\lambda] /(p(\lambda))$ and as such has a basis $\left\{x_{\alpha}\right\}_{\alpha}$ over $K$. Now 1 , $\lambda, \cdots, \lambda^{m+1}$ modulo $p(\lambda)$ form a basis for $K$ over $F$ and so well known 
vector space arguments show $\left\{x_{\alpha} \lambda^{j}\right\}_{\alpha, j}$ to be a basis of $V_{1}$ over $F$. Now $x_{\alpha} \lambda^{j}=x_{\alpha} D^{j}$ and so $\left\{x_{\alpha} D^{j}\right\}_{\alpha, j}$ is a basis of $V_{1}$ over $F$. To simplify the notation write $x_{\alpha}=x(\alpha, 1)$ and choose inductively (by the divisibility hypothesis) $x(\alpha, k+1)$ in $V_{k+1}$ with $x(\alpha, k+1) p(D)=x(\alpha, k)$.

The vectors $\left\{x(\alpha, k) D^{j}\right\}_{\alpha, j, k}$ are linearly independent over $F$. For if

$$
\sum_{\alpha} \sum_{k=1}^{n+1} \sum_{j=0}^{m-1} \beta(\alpha, k, j) x(\alpha, k) D^{j}=0
$$

apply $p(D)^{n}$ to (2) to obtain

$$
\sum_{\alpha} \sum_{j=0}^{m-1} \beta(\alpha, n+1, j) x(\alpha, 1) D^{j}=0 .
$$

By the choice of $x(\alpha, 1)$, relation (3) yields $\beta(\alpha, n+1, j)=0$ and an obvious induction establishes that all $\beta(\alpha, k, j)=0$. To see that the chosen vectors span $V$, the argument proceeds from $V_{k}$ to $V_{k+1}$. In order to avoid an excessive amount of notation the step from $V_{1}$ to $V_{2}$ will be indicated. If $x \in V_{2}, x p \in V_{1}$ so that $x p=\sum_{\alpha, j} \beta(\alpha, j) x(\alpha, 1) D^{j}$; let $y=\sum_{\alpha, j} \beta(\alpha, j) x(\alpha, 2) D^{j}$ and observe that $z=x-y$ lies in $V_{1}$ since $y p=x p$. Thus $z=\sum_{\alpha, j} \gamma(\alpha, j) x(\alpha, 1) D^{j}$ and $x=y+z$ $=\sum_{\alpha, j} \beta(\alpha, j) x(\alpha, 2) D^{j}+\sum_{\alpha, j} \gamma(\alpha, j) x(\alpha, 1) D^{j}$.

To conclude the proof of Theorem $1, T$ is explicitly constructed in terms of the basis $\left\{x(\alpha, k) D^{j}\right\}$ of Lemma 3. Define

$$
\begin{aligned}
& x(\alpha, k) T=x(\alpha, k+1), \\
& x(\alpha, k) D T=x(\alpha, k+1) D-x(\alpha, k), \\
& x(\alpha, k) D^{2} T=x(\alpha, k+1) D^{2}-2 x(\alpha, k) D, \\
& \quad \vdots \\
& x(\alpha, \dot{k}) D^{m-1} T=x(\alpha, k+1) D^{m-1}-(m-1) x(\alpha, k) D^{m-2} .
\end{aligned}
$$

It only remains to establish $[T, D]=I$. The calculation is as follows:

$$
\begin{aligned}
x(\alpha, k) D^{j} T D & =\left[x(\alpha, k+1) D^{j}-j x(\alpha, k) D^{j-1}\right] D \\
& =x(\alpha, k+1) D^{j+1}-j x(\alpha, k) D^{j}
\end{aligned}
$$

and

$$
x(\alpha, k) D^{j}(D T)=x(\alpha, k+1) D^{j+1}-(j+1) x(\alpha, k) D^{j} .
$$

Upon differencing these two results one obtains

$$
x(\alpha, k) D^{j}(T D-D T)=x(\alpha, k) D^{j}
$$

which is exactly the desired result. It should be remarked that these 
calculations are valid when $j=m-1$ since in this case $D^{j+1}=D^{m}$ is expressible as a linear combination of lower powers of $D$.

3. Necessity for characteristic zero. In this section $F$ will designate a field of characteristic 0 and $V$ will be a vector space over $F$ with two linear transformations, $D$ and $T$, satisfying $[T, D]=I$. Moreover it is assumed that $V$ is locally algebraic with respect to $D$. Again the problem is reduced to the primary case by Lemmas 1 and 2 , but it is necessary to show that the primary components of $V$ are invariant under $T$ before the reduction can be made.

LEMмA 4. Let $T, D$ be linear transformations of $V$ satisfying $[T, D]$ $=I$. For any polynomial $f(\lambda)$ in $F[\lambda]=A$

$$
\begin{aligned}
T D^{k} & =D^{k} T+k D^{k-1}, \\
T f(D) & =f(D) T+f^{\prime}(D)
\end{aligned}
$$

where $f^{\prime}(\lambda)$ designates the usual derivative of $f(\lambda)$. Furthermore, if $V_{p}$ is a primary component of $V$ (relative to $D$ ) then $V_{p}$ is $T$-invariant.

The first relation in (5) is readily established by induction and the second is an immediate consequence thereof. To see that $V_{p}$ is $T$ invariant observe first that $V_{p}$ is $D$-invariant. Then for any $x \in V_{p}$ let $x(p(D))^{r}=0$ and note $(x T)(p(D))^{r}=x(p(D))^{r} T+x\left(p(D)^{r}\right)^{\prime}$ $=x(p(D))^{r} T+r x(p(D))^{r-1} p^{\prime}(D)=r x(p(D))^{r-1} p^{\prime}(D)$ which lies in $V_{p}$ since $V_{p}$ is $D$-invariant.

For the remainder of this section it is assumed that $V$ is a primary $A$-module such that $[T, D]=I$. Define the subspaces $V_{k}$ by (1) again. Then the following lemma holds [3].

LeMmA 5. If every $y$ in $V_{1}$ has the property that for each integer $k \geqq 1$, there is an $x$ in $V$ with $y=x(p(D))^{k}$ then $V$ is divisible.

To simplify the following calculations, the notation $x f, x T f, T^{k} f$, etc., is used in lieu of $x f(D), x T f(D), T^{k} f(D)$, etc. There are several steps which culminate with the verification of the hypothesis of Lemma 5. These steps are listed below where $(f, g)=1$ signifies as usual that the polynomials $f(\lambda)$ and $g(\lambda)$ are relatively prime.

(a) If $(f, p)=1$ and $y \in V$ there is an $x \in V$ with $y=x f$. For if $y \in V_{k}$ write $f g+h p^{k}=1$ so that $y f g+y p^{k} h=y$; the desired conclusion follows with the choice $x=y g$.

(b) If $y \in V_{k}, y f=z p^{n}$ where $(f, p)=1$ then there is an $x \in V$ with $y=x p^{n}$. Again write $f g+p^{k} h=1$ so that $z g p^{n}=z p^{n} g=y g f=y\left[1-p^{k} h\right]$ $=y-y p^{k} h=y$. For the choice $x=z g$ the conclusion $x p^{n}=y$ follows. 
(c) An easy induction establishes the commutativity relation

$$
T^{m} f=\sum_{k=0}^{m}\left(\begin{array}{c}
m \\
k
\end{array}\right) f^{(k)} T^{m-k}
$$

where $f^{(k)}$ designates the $k$ th derivative of $f$.

(d) The following known result is easily established by induction.

$$
\begin{aligned}
\left(p^{n}\right)^{(k)}= & n(n-1) \cdots(n-k+1) p^{n-k}\left(p^{\prime}\right)^{k} \\
& +p^{n-k+1} f_{k}\left(p, p^{\prime}, \cdots, p^{(k)}\right)
\end{aligned}
$$

where $f_{k}\left(p, p^{\prime}, \cdots, p^{(k)}\right)$ is an integral polynomial in $p, p^{\prime}, \cdots, p^{(k)}$.

(e) For each $y$ in $V_{1}$ there is $x$ in $V$ with $y=x p^{n}$. For let $z=y T^{n}$ and compute

$$
z p^{n}=y T^{n} p^{n}=y \sum_{k=0}^{n}\left(\begin{array}{l}
n \\
k
\end{array}\right)\left(p^{n}\right)^{(k)} T^{n-k}
$$

by (c). By (d) above

$$
y\left(p^{n}\right)^{(k)}=y\left[k !\left(\begin{array}{l}
n \\
k
\end{array}\right) p^{n-k}\left(p^{\prime}\right)^{k}+p^{n-k+1} f_{k}\left(p, p^{\prime}, \cdots, p^{(k)}\right)\right]=0
$$

for $k<n$ since $y p=0$. Thus

$$
z p^{n}=y\left(p^{n}\right)^{(n)}=y\left[n !\left(p^{\prime}\right)^{n}+p f_{n}\left(p, p^{\prime}, \cdots, p^{(n)}\right)\right]=y\left[n !\left(p^{\prime}\right)^{n}\right] .
$$

Since the field is of characteristic 0 , the irreducibility of $p(\lambda)$ ensures $\left(p, p^{\prime}\right)=1$; also $n ! \neq 0$ in $F$ and so $\left(n ! p^{\prime}, p\right)=1$ and the conclusion follows immediately from (b). The hypothesis of Lemma 5 has been established and the proof of Theorem 2 is complete.

A counterexample for finite characteristic is readily given. For example if $F=\mathrm{GF}(3)$ and $V$ has basis $x_{1}, x_{2}, x_{3}$ over $F$ define $T$ by $x_{1} T=x_{2}, x_{2} T=x_{3}, x_{3} T=0$ and $D$ by $x_{1} D=0, x_{2} D=x_{1}$ and $x_{3} D=2 x_{2}$. An easy check shows $T D-D T=I$ and $D$ is surely singular. It is clear that if $V$ were divisible as an $A$-module, $D$ would have to map $V$ onto itself and so $V$ cannot be divisible. Closely related to these results is a result of Albert and Muckenhoupt [1] which states that if $S$ is a linear transformation of the finite dimensional vector space $V$ over $F$ it is a commutator, i.e., $S=T U-U T$ for linear transformations $U$, $T$ of $V$ if and only if Trace $S=0$.

4. Results of Mikusinski and Geer. In [2] Mr. Geer gave a generalization of Mikusinski's result. Using Theorem 1 it is easy to prove a result which includes both of their results and is stated as 
THEOREM 3. Let $D$ be a locally algebraic linear operator on $V$ satisfying (i) for each irreducible $p(\lambda)$ in $A$ the kernel of $p(D)$ is finite dimensional,

(ii) for each irreducible $p(\lambda)$, dim $\operatorname{ker} p^{n}=n(\operatorname{dim} \operatorname{ker} p)$. Then $a$ linear operator $T$ on $V$ exists with $[T, D]=I$.

The only hypothesis of Theorem 1 that must be verified is the divisibility condition and by Lemmas 1 and 2 it suffices to verify this condition for each primary component of $V$. Obviously the restriction of $D$ to a primary component also satisfies (i) and (ii) and so it may be assumed that $V$ is primary for some prime $p=p(\lambda)$. The subspaces $V_{k}$ are again defined by (1) so that $V_{k+1} p \subset V_{k}$ and the kernel of $p(D) \mid V_{k+1}$ is clearly $V_{1}$ so $V_{k+1} / V_{1}$ is $A$-isomorphic to $V_{k} p$. Thus $\operatorname{dim} V_{k+1}-\operatorname{dim} V_{1}=\operatorname{dim} V_{k+1} p$ but by (ii) $\operatorname{dim} V_{k+1}$ $=(k+1)\left(\operatorname{dim} V_{1}\right)$ and so $\operatorname{dim} V_{k+1} p=k\left(\operatorname{dim} V_{1}\right)$ which is $\operatorname{dim} V_{k}$ by (ii). Therefore, $V_{k+1} p \subset V_{k}$ together with the dimension count given shows $V_{k+1} p=V_{k}$ and the condition of Lemma 5 is verified and $V$ is divisible as desired.

For completeness the converse of Mikusinski is deduced from Theorem 2 in the following form.

Theorem 4. Let $D$ be a linear operator on the vector space $V$ over the field $F$ of characteristic 0 such that $D$ is locally algebraic on $V$. Suppose that for each irreducible $p(\lambda)$ in $A=F[\lambda], \operatorname{dim} \operatorname{ker} p$ is finite and suppose that a linear operator $T$ of $V$ exists with $[T, D]=I$, then condition II is satisfied and $\operatorname{dim} \operatorname{Ker} f(D)$ is finite for every $f(\lambda)$ of positive degree.

By Theorem 2, $V$ must be a divisible $A$-module and so is each primary component of $V$ by Lemmas 1 and 2. If $S$ is a primary component, let $S_{k}=\operatorname{Ker} p^{k}$ so that the divisibility property of $S$ ensures $S_{k+1} p=S_{k}$. Since $\operatorname{Ker} p(D) \mid S_{k+1}=S_{1}$ the isomorphism theorem yields $S_{k+1} / S_{1} A$-isomorphic to $S_{k+1} p=S_{k}$. Thus $\operatorname{dim} S_{k+1}=\operatorname{dim} S_{k}+\operatorname{dim} S_{1}$ and $\operatorname{dim} S_{1}$ is finite by hypothesis; an obvious induction argument establishes $\operatorname{dim} S_{k+1}=(k+1) \operatorname{dim} S_{1}=(k+1) \operatorname{dim} \operatorname{Ker} p$ as desired. Next, observe that if $f(\lambda), g(\lambda)$ are relatively prime then $\operatorname{Ker} f g$ $=\operatorname{Ker} f+\operatorname{Ker} g$. For surely $\operatorname{Ker} f+\operatorname{Ker} g \subset \operatorname{Ker} f g$ holds; consequently, write $1=f h+g k$ for $h, k$ in $A$ so that $x$ in Ker $f g$ can be written as $x=x f h+x g k$ where $x f h \in \operatorname{Ker} g$ and $x g k \in \operatorname{Ker} f$ is obvious. This shows $\operatorname{Ker} f+\operatorname{Ker} g=\operatorname{Ker} f g$; finally if $x \in(\operatorname{Ker} f) \cap(\operatorname{Ker} g)$ then $x=x f h+x g k=0$ and so the sum is direct. The desired conclusion is now an obvious consequence of the preceding results and the unique factorization in $A$. 


\section{REFERENCES}

1. A. Albert, and F. Muckenhoupt, On matrices of trace 0, Michigan Math. J. 4 (1957), 1-3.

2. James Geer, An extension of Mikusinski's algebraic theory of differential equations, Masters Thesis, Univ. of Virginia, Charlottesville, 1964.

3. I. Kaplansky, Infinite Abelian groups, Univ. of Michigan Press, Ann Arbor, Mich., 1965.

4. J. Mikusinski, Sur les solutions lineairement independantes des equations differentielles a coefficients constants, Studia Math. 16 (1957), 41-47.

5. —_, Sur l'espace lineaire avec derivation, Studia Math. 16 (1957), 113-123.

6. R. Sikorski, On Mikusinski's algebraical theory of differential equations, Studia Math. 16 (1957), 230-36.

UNIVERSITY OF VIRGINIA 